\title{
Automated and Shaped-Controlled Liquid STEM Nanolithography
}

\author{
Raymond R. Unocic ${ }^{1,2}$, Andrew R. Lupini ${ }^{1,3}$, Albina Y. Borisevich ${ }^{1,3}$, Sergei V. Kalinin ${ }^{1,2}$, and \\ Stephen Jesse ${ }^{1,2}$ \\ ${ }^{1 .}$ Institute for Functional Imaging of Materials, Oak Ridge National Laboratory, Oak Ridge, TN 37831 \\ 2. Center for Nanophase Materials Sciences, Oak Ridge National Laboratory, Oak Ridge, TN 37831 \\ 3. Materials Science \& Technology Division, Oak Ridge National Laboratory, Oak Ridge, TN 37831
}

There is a range of scanning probe and electron beam nanolithography platforms currently available for producing nano-scaled structures with complex shapes and chemistries from solid or gaseous phase precursors [1-2]. Recent studies have shown that the same concept can be applied to liquid phase precursors using liquid cells and electron beams with manual manipulation [3-4]. In the present work, an automated electron beam control system has been developed to precisely control the position and residence time of the STEM probe from a $\mathrm{C}_{\mathrm{s}}$ aberration-corrected FEI Titan STEM operating at $300 \mathrm{kV}$. The position of the STEM probe is controlled by applying bias waveforms to the X- and Y- STEM scan coils such that the focused STEM probe can be positioned in the X-Y plane either in a fixed position with a pre-defined dwell time or rastered in the X-Y plane with a variable translational velocity.

This system has been used for nanolithographic patterning of nm-scale 3D structures from liquid phase precursor solutions. An in situ liquid cell TEM holder was used to encapsulate a $10 \mu \mathrm{M}$ $\mathrm{H}_{2} \mathrm{PdCl}_{4}$ aqueous solution between two $50 \mathrm{~nm}$ thick silicon nitride membranes. Radiolysis occurs when the electron beam interacts with the solution, forming complex radical species [5]. As a result, aqueous electrons $\left(\mathrm{e}_{\mathrm{aq}}{ }^{-}\right)$are generated in the liquid, which effectively act as a reducing agent to reduce metallic $\mathrm{Pd}$ from the $\mathrm{H}_{2} \mathrm{PdCl}_{4}$ complex [6]. Thus, the principals of radiation chemistry within closed liquid cells combined with automated electron beam control can be exploited to nanolithographically-pattern structures of metallic Pd.

The Institute of Functional Imaging of Materials (IFIM) acronym was used to demonstrate the concept of liquid STEM nanolithography. The focused STEM probe was rastered through the liquid cell containing the $10 \mu \mathrm{M} \mathrm{H} \mathrm{H}_{2} \mathrm{PdCl}_{4}$ growth solution and $\mathrm{Pd}$ metal was reduced forming $\mathrm{Pd}$ nanocrystals directly onto the silicon nitride membrane and within the liquid cell through a nucleation and growth mechanism. An annular dark field (ADF) STEM image of the patterned "IFIM" text is shown in Figure 1a. As a result of chemically sensitive ADF STEM imaging, whereby intensity is proportional to the atomic number and thickness, the nanolithographically patterned $\mathrm{Pd}$ has a higher intensity when compared against the lower atomic number background of the silicon nitride membranes and the $\mathrm{H}_{2} \mathrm{PdCl}_{4}$ growth solution and therefore the electron beam reduced $\mathrm{Pd}$ structures can readily be characterized following patterning. It is clear by the size-scale and legibility of the IFIM letters shown in Figure 1a, that the nanolithography technique can be used to precisely control the position of the STEM probe and resultant Pd crystal deposition. Corresponding higher magnification images shown in Figures $1 \mathrm{~b}-\mathrm{d}$, reveal that the pattern is composed of Pd nanocrystals having individual crystal sizes on the order of tens of nanometers with the exception of a few larger crystals (Figure $1 \mathrm{~b}$ ). It is interesting to note that the Pd nanocrystals are primarily confined within the local irradiated region of the IFIM pattern, e.g., there is limited nucleation and growth of Pd outside of this area. For liquid STEM nanolithography, precision-controlled nanosculpting is 
governed by the interplay between electron dose, electron beam exposure time, diffusivity of $\mathrm{e}_{\mathrm{aq}}{ }^{-}$, and nucleation and growth kinetics of the electron beam deposited crystals [7].

References:

[1] R Garcia et al, Nature Nanotechnology 9 (2014) p. 577-587.

[2] I Utke et al, Journal of Vacuum Science and Technology B 26 (2008) p. 1197-1276.

[3] JM Grogan et al, Nano Letters 15 (2014) p. 359-364.

[4] MWP van de Put et al, Small 11 (2015) p. 585-590.

[5] NM Schneider et al, Journal of Physical Chemistry C 118 (2014) p. 22373-22382.

[6] KL Jungjohann et al, Nano Letters 13 (2013) p. 2967-2970.

[7] Research supported by Oak Ridge National Laboratory's Center for Nanophase Materials Sciences (CNMS), which is a U.S. Department of Energy, Office of Science User Facility. (RRU, SVK, and SJ) Additional support provided by the Division of Materials Sciences and Engineering Division, Office of Basic Energy Sciences, U.S. DOE. (ARL and AYB)

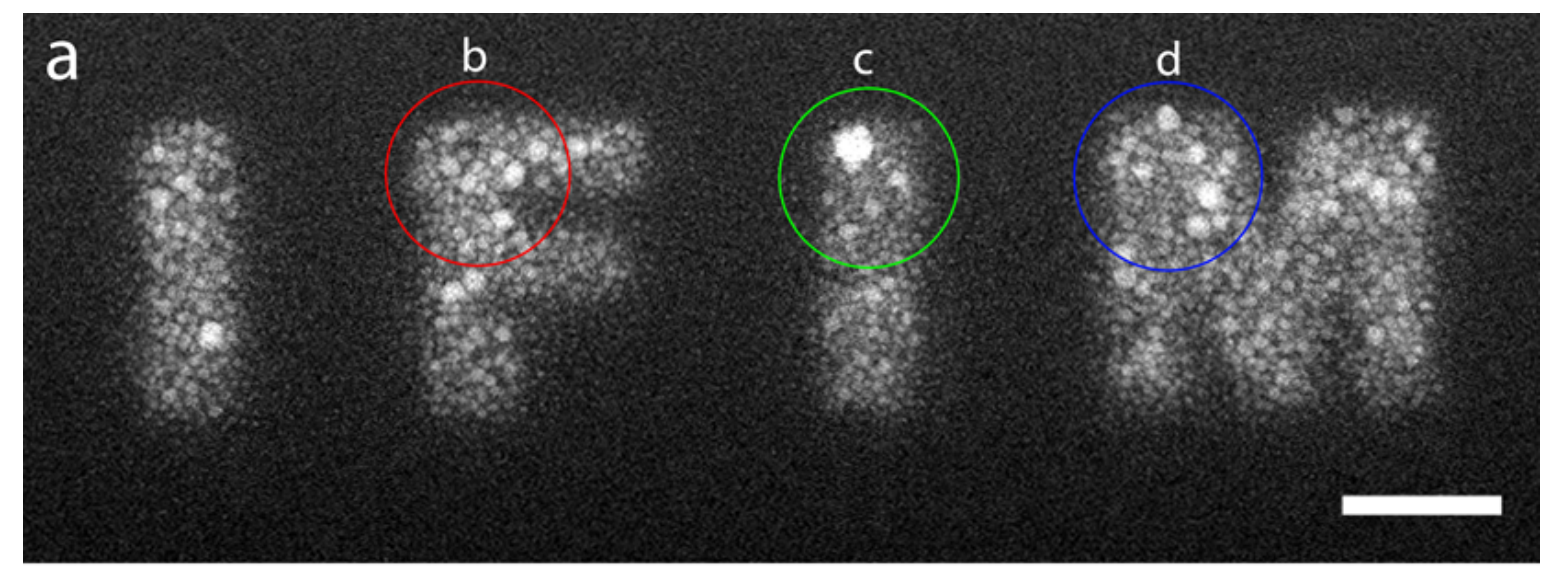

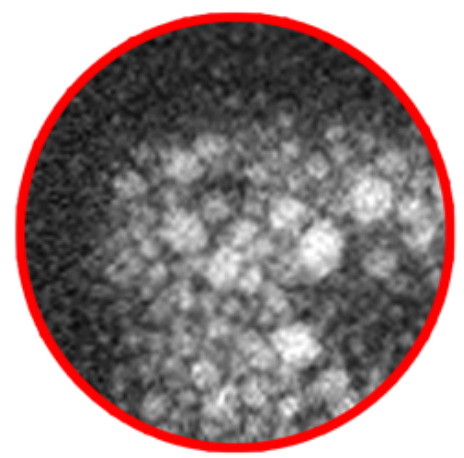

b

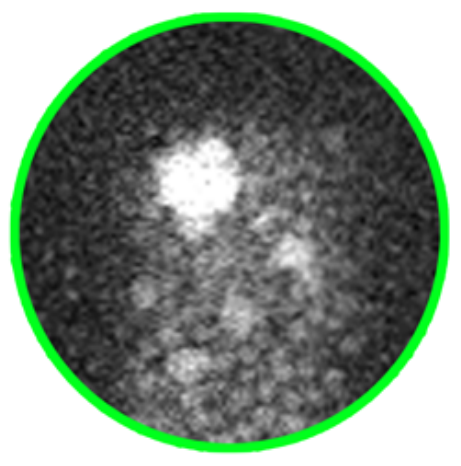

C

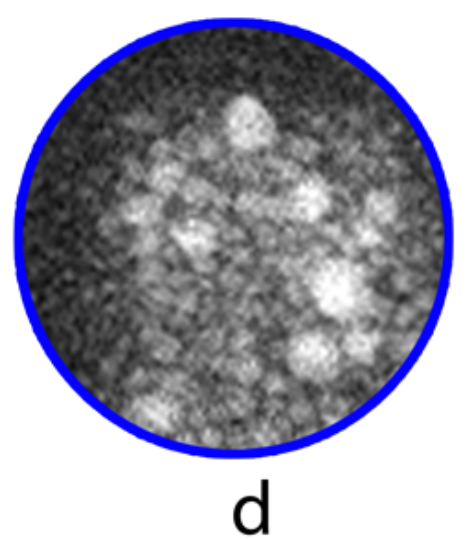

Figure 1. a) ADF STEM image showing the "IFIM" text nanolithographically patterned within a STEM liquid cell using a $10 \mu \mathrm{M} \mathrm{H}_{2} \mathrm{PdCl}_{4}$ precursor growth solution. Scale bar is $500 \mathrm{~nm}$. b-d) Higher magnification ADF STEM images from several regions of the pattern showing the detailed nanocrystalline nature of the deposited $\mathrm{Pd}$. 